\title{
CARACTERISTICAS DE LA ENFERMEDAD PULMONAR OBSTRUCTIVA CRONICA EN FUMADORES Y EX FUMADORES ATENDIDOS EN UN HOSPITAL DE LA PROVINCIA DE ICA, PERÚ
}

\author{
Ethel Vidalón-Soldevilla ${ }^{1, a}$, Ysabel Ventura-Fernández ${ }^{2, b}$, Rafael Torres-Godomar ${ }^{1, c}$.
}

1. Facultad de Medicina, Universidad Nacional San Luis Gonzaga. Ica, Perú.

2. Ministerio Público, División Médico Legal I de Pisco. Ica, Perú.

a. Médico Internista. b. Médico Cirujano. c. Neumólogo

\section{RESUMEN}

Objetivo: Determinar las características de la Enfermedad Pulmonar Obstructiva Crónica (EPOC) en fumadores y ex fumadores atendidos en un hospital de la provincia de Ica, Perú. Material y métodos: Estudio descriptivo, retrospectivo, observacional; mediante un muestreo no probabilístico por conveniencia se seleccionaron 70 Fumadores y 70 Ex fumadores se realizo una entrevista directa, recogiendo las características clínicas así como la aplicación del examen espirométrico. Resultados: En los fumadores el $60 \%$ fueron masculinos; en el grupo de Ex fumadores, se observo que el $54 \%$ era femenino, el grado de EPOC fue severo en $55 \%$ de fumadores y moderado en $54 \%$ de ex fumadores. Según el motivo de hospitalización, en fumadores el $56 \%$ fue por exacerbación de tos crónica y en ex fumadores bronquitis crónica y disnea con $36 \%, 30 \%$ respectivamente. Conclusiones: Más del 50\% de fumadores presentó un grado de severo de EPOC y en ex fumadores el $54 \%$ presentó un grado moderado de EPOC, en ambos grupos las hospitalizaciones por diferentes patologías, presentó en común exacerbación de tos crónica, bronquitis crónica y disnea; el índice tabáquico es de severo a moderado.

( Rev. méd. panacea 2011; 1:67 - 71 ).

Palabras claves: Fumar, Tos, Espirometria.. (fuente: DeCS BIREME).

\section{CHARACTERISTICS OF CHRONIC OBSTRUCTIVE PULMONARY DISEASE IN SMOKERS AND FORMER SMOKERS AT A HOSPITAL IN THE PROVINCE OF ICA, PERU}

\begin{abstract}
Objetive: To determine the characteristics of Cronic Obstructive Pulmonary Disease (COPD) in smokers and former smokers treated at a Hospital in Ica province, Peru. Materials and Methods: A descriptive, retrospective, observational study. Trough a non-probability sampling by convenience were selected 70 smokers and 70 former smokers. A direct interview was realized, collecting clinical characteristics as well as applying spirometric examination. Results: The $60 \%$ were male smokers; there were $54 \%$ of female former smokers. A severe degree of chronic obstructive pulmonary disease (COPD) in 55\% of smokers and former smokers $54 \%$ had a moderate degree. Hospitalizations experienced, $56 \%$ of smokers presenting chronic cough, in former smokers: $36 \%$ due to cronic bronchitis and $30 \%$ with dysnea. Conclusions: more than $50 \%$ of smokers presented a severe degree of COPD and $54 \%$ of former smokers presented a moderate degree of COPD. In both groups, hospitalizations had different pathologies, having in common chronic cough, chronic bronchitis and dysnea, smoking rate is moderate to severe. (Rev. méd. panacea 2011; 1:67 - 71).
\end{abstract}

Key words: Smoke, Cough, Espirometry. (source: MeSH NLM).

\section{INTRODUCCIÓN}

La Enfermedad Pulmonar Obstructiva Crónica (EPOC) es un problema importante para la salud pública siendo una de las principales causas de morbilidad entre las enfermedades crónicas en el mundo, y la cuarta causa de muerte a escala mundial, con una prevalencia y mortalidad que tienden a incrementarse durante las próximas décadas $(1-11)$
La EPOC es una enfermedad progresiva e incapacitante, se extiende en forma alarmante en la sociedad haciéndose más evidente en las grandes ciudades, relacionado con múltiples factores como el consumo de tabaco, polución, estilos de vida, ocupacional, agregándose además el factor genético $^{(12-20)}$

Esta enfermedad se relaciona con el hábito de fumar, motivo del presente estudio cuyo objetivo fue determinar las características de la Enfermedad Pulmonar Obstructiva 
Crónica (EPOC) en fumadores y ex fumadores atendidos en un hospital de la provincia de Ica, Perú

\section{MATERIAL Y MÉTODOS}

Se realizó una investigación de tipo descriptivo, observacional y transversal.

Se realizo un muestreo no probabilístico, por conveniencia consideró como referencia a 720 pacientes fumadores y ex fumadores con diagnóstico de EPOC que acudieron al Servicio de Neumología del Hospital "Felix Torrealva Gutierrez" de EsSalud Ica durante los meses de junio del 2005 a junio del 2006, siendo incluidos 140 pacientes para el estudio, 70 Fumadores y 70 Ex fumadores.

Se considero como criterios de inclusión: fumadores y ex fumadores con EPOC que acudieron al servicio de Neumología del Hospital "Félix Torrealva Gutiérrez" y aceptaron participar en el estudio, se excluyeron pacientes con diagnóstico de EPOC que no eran fumadores o ex fumadores, los que acudieron a otro hospital, pacientes con diagnóstico de neoplasia pulmonar benigna o maligna, pacientes con espirometrías fallidas y los que no aceptaron ingresar al estudio.

Se utilizo una ficha de recolección de datos, elaborada por el investigador y validada en 30 pacientes del hospital "Félix Torrealva Gutiérrez", para luego ser utilizada en la muestra seleccionada, consignándose los datos de: filiación, edad, hábito de fumar, ocupación, sexo, evaluación espirométrica.

Para medir el índice tabáquico se considero como fumador severo a quienes consumían más de 20 paquetes/año; fumador moderado entre 10 y 20 paquetes/año y fumador leve menos de 10 paquetes/ año.

Utilizando el programa Epilnfo 2000; se realizo un análisis univariado y bivariado con un IC 95\% considerando un $p<0,05$ con estadísticamente significativo.

\section{RESULTADOS}

Según la variable sexo se observó: en los fumadores el $60 \%$ fueron masculinos y el $40 \%$ femenino; en el grupo de ex fumadores, se observo que el $54 \%$ era femenino y $46 \%$ masculino, teniendo un valor de $p<0,12$.

Según la edad en ambos grupos, se observó que: en el grupo de fumadores, el $41 \%$ se hallaba entre los 26 a 60 años de edad en el grupo de los ex fumadores el $52 \%$ se hallaba en el grupo de $>$ de 60 años $(p<0,06)$.

Respecto al estado civil: en el grupo de fumadores el $76 \%$ se encontraba casado y el $24 \%$ soltero y en el grupo de ex fumadores el $61 \%$ casado y el $39 \%$ soltero $(p<0,06)$.
Según el Índice tabáquico: en el grupo de fumadores el $50 \%$ era fumador severo y el $30 \%$ fumador moderado; en el grupo de ex fumadores el $47 \%$ era fumador moderado y el $30 \%$ fumador severo $(p<0,042)$.

Tabla 1. Características de la Enfermedad Pulmonar Obstructiva Crónica en fumadores y ex fumadores atendidos en un hospital de la provincia de Ica, Perú.

\begin{tabular}{|c|c|c|c|c|c|}
\hline \multirow{2}{*}{ Características } & \multicolumn{2}{|c|}{ Fumadores } & \multicolumn{2}{|c|}{$\begin{array}{c}\text { Ex } \\
\text { Fumadores }\end{array}$} & \multirow{2}{*}{$p$} \\
\hline & $\mathbf{n}$ & $(\%)$ & $\mathbf{n}$ & $(\%)$ & \\
\hline \multicolumn{6}{|l|}{ Sexo } \\
\hline Hombre & 42 & $(60)$ & 32 & $(46)$ & 0,12 \\
\hline Mujer & 28 & 40 & 38 & $(54)$ & \\
\hline \multicolumn{6}{|l|}{ Edad } \\
\hline 15 a 26 años & 18 & (26) & 10 & (14) & 0,06 \\
\hline 26 a 60 años & 29 & (41) & 24 & (34) & \\
\hline > 60 años & 23 & (33) & 36 & (52) & \\
\hline \multicolumn{6}{|l|}{ Estado civil } \\
\hline Soltero & 17 & (24) & 27 & (39) & 0,06 \\
\hline Casado & 53 & (76) & 43 & (61) & \\
\hline \multicolumn{6}{|l|}{ Índice tabáquico } \\
\hline Fumador Leve & 14 & (20) & 16 & (23) & 0,04 \\
\hline Fumador Moderado & 21 & (30) & 33 & $(47)$ & \\
\hline Fumador Severo & 35 & $(50)$ & 21 & $(30)$ & \\
\hline \multicolumn{6}{|l|}{ Tiempo de consumo } \\
\hline$<10$ años & 15 & $(21)$ & 27 & (39) & 0,04 \\
\hline > 10 años & 55 & (79) & 43 & (61) & \\
\hline \multicolumn{6}{|l|}{ Intento de abandono } \\
\hline$<5$ veces & 54 & $(77)$ & 29 & $(41)$ & 0,01 \\
\hline$>5$ veces & 16 & (23) & 41 & (59) & \\
\hline
\end{tabular}

Según el tiempo de consumo de cigarrillos: en el grupo de fumadores, el $79 \%$ tenía más de 10 años de consumo y el $21 \%$ menos de 10 años; en el grupo de ex fumadores el $61 \%$ presentaba más de 10 años de consumo teniendo un valor estadísticamente significativo $(p<0,042)$.

Considerando el número de intentos de abandono al cigarrillo se halló: en el grupo de fumadores el $77 \%$ lo intentó menos de 5 veces; en el grupo de ex fumadores se halló que el $59 \%$ intento abandonarlo más de 5 veces con un valor de $p<0,01$.

Analizado el motivo de hospitalización se encontró que en el grupo de fumadores el $56 \%$ ingresó presentando tos crónica y $25 \%$ bronquitis crónica y mientras que en el grupo de ex fumadores el $36 \%$ se hospitalizó por presentar bronquitis crónica, y $34 \%$ tos crónica $(p<0,074)$ (Figura 1). 
Visto el grado de intensidad de la EPOC en el grupo de fumadores se observó que el $55 \%$ presentó un grado severo de EPOC y en el grupo de ex fumadores el $54 \%$ presentó un grado moderado $(p<0,01)$ (Figura 2).

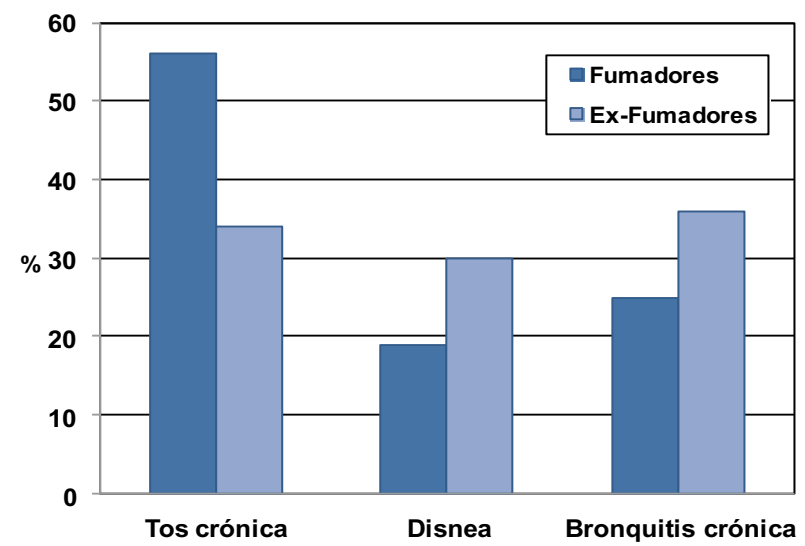

Figura 1. Presentación de la Enfermedad Pulmonar Obstructiva Crónica en fumadores y ex Fumadores atendidos en un hospital de la provincia de Ica.

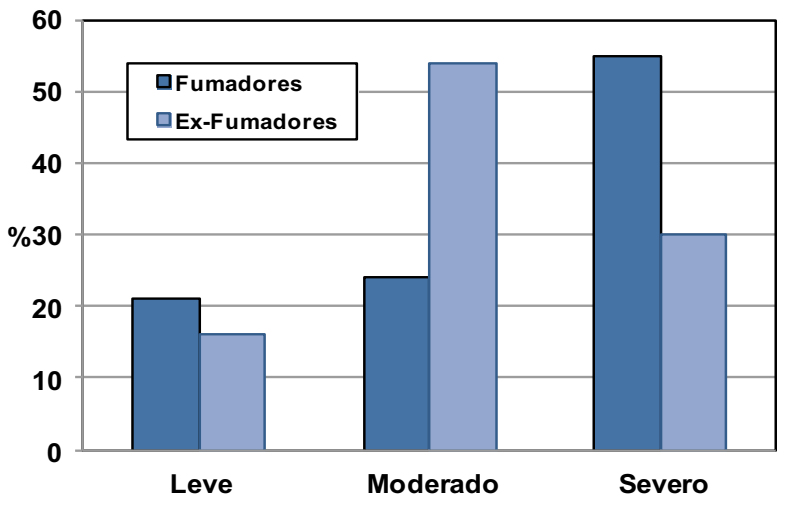

Figura 2. Grados de Enfermedad Pulmonar Obstructiva Cronica en fumadores y ex fumadores atendidos en un hospital de la provincia de Ica.

\section{DISCUSIÓN}

En este trabajo no se encontró diferencia significativa en cuanto al género, sin embargo los datos bibliográficos indican que la prevalencia media del tabaquismo en América Latina es del $37 \%$ en los varones y del $20 \%$ en las mujeres. No obstante, hay una gran variabilidad, de manera que en países como Uruguay, México, Chile y Perú pueden encontrarse cifras entre el 40,3 y el $66 \%$ en segmentos de población urbana ${ }^{(20)}$.

Sobre la edad se observó que en fumadores el mayor porcentaje perteneció a la etapa adulta, en la práctica de este hábito Vs un $52 \%$ de exfumadores en la etapa de adulto mayor; según el grupo de trabajo de la Asociación Latinoamericana de Tórax: "Un aspecto de vital importancia es la precocidad en el inicio del consumo, ya que la mayoría de los fumadores se inician en el hábito entre 13 y 15 años de edad. En Guatemala fuma el 25\% de adolescentes de 15 años de edad y en Uruguay el $30 \%$. En Brasil fuman el $4,4 \%$ de adolescentes entre 12 y 17 años de sexo masculino y el $3 \%$ de las jóvenes de la misma edad" 5,20 .

Se confirmó la asociación de fumar con el vivir con una pareja, demostrado en algunos estudios y en el nuestro, otros estudios no hacen referencia al estado civil.

El índice tabáquico de severo a moderado, predominó en el grupo de fumadores en nuestro trabajo, corroborando la tendencia del aumento de este hábito en la sociedad manifestado en otros estudios.

Nosotros observamos más años de consumo en pacientes fumadores activos, corroborado por la literatura revisada ${ }^{15}$.

Se encontró también una fuerte tendencia y voluntad de abandonar este hábito de fumar en el grupo de ex fumadores, mas no en el grupo de fumadores cuyos intentos de abandono no se acentúan. El abandono del hábito de fumar, además de ser la medida más eficaz y rentable para reducir el riesgo de presentar EPOC y detener su progresión, se asocia con una disminución del número de accidentes cardíacos agudos que se presentan y con una mejoría de las lesiones vasculares arterioscleróticas que puedan existir ${ }^{20-25}$. No obstante, conviene señalar que, de acuerdo con el estudio de Sin y Man, la relación existente entre los cambios que se suceden en el volumen espiratorio forzado en el primer segundo (FEV1) y la aparición de complicaciones aterotrombóticas se da con independencia de los efectos del tabaco ${ }^{25}$.

No se encontró diferencia estadísticamente significativa entre fumadores y exfumadores para el motivo de hospitalización, pero si se observó asociación de ambos grupos con el tener: tos, bronquitis y disnea, características clínicas corroboradas por la Asociación Latinoamericana de Tórax ${ }^{5}$.

Nuestro trabajo determinó una fuerte asociación entre el fumar y presentar grados moderados y severos de EPOC, confirmando los hallazgos de otros estudios internacionales ${ }^{5,18}$.

En conclusión, más del $50 \%$ de fumadores presentó un grado severo de Enfermedad Pulmonar Obstructiva Crónica (EPOC) y en exfumadores el $54 \%$ presentó un grado moderado de EPOC. En ambos grupos las hospitalizaciones, por diferentes patologías, presentaron en común: exacerbación de tos crónica, bronquitis crónica y disnea. El índice tabáquico es de severo a moderado sobre todo en el grupo de fumadores, corroborándose la tendencia y gravedad de la enfermedad asociada al mayor consumo de tabaco manifestado en otros estudios. En más del $50 \%$ del grupo de fumadores la intención de abandonar el consumo de tabaco fue muy baja, mientras que un 
menor número de ellos intento abandonarlo en más de 5 oportunidades. El grupo de ex fumadores intentó abandonar el hábito de fumar más de 5 veces y en menor proporción menos de 5 veces antes del abandono definitivo. El $79 \%$ de fumadores llevaba más de 10 años de consumo, mientras que en el grupo de exfumadores el $61 \%$ presentó más de 10 años de consumo, antes del cese definitivo de fumar.

Deben desarrollarse estrategias, a través de los equipos básicos de salud, para que en la práctica asistencial se brinden consejerías a favor de disminuir el consumo de cigarrillos en los pacientes que acuden al Hospital "Félix Torrealva Gutiérrez" de Ica, difundiendo también la clínica y el diagnóstico de la Enfermedad Pulmonar Obstructiva Crónica (EPOC).

Igualmente, las estrategias deben ser compartidas con los profesionales responsables de la Atención Primaria Salud para reducir el porcentaje de EPOC, permitiendo un diagnóstico y tratamiento en los estadios precoces de la enfermedad, contribuyendo de esta manera a una mayor prevención de la misma.

\section{Correspondencia:}

Ethel Vidalon Sodevila.

Correo electrónico: esv2706@jotmail.com

Teléfono: 056-225262

\section{REFERENCIAS BIBLIOGRÁFICAS}

1. Escarrabil. Enfermedad Pulmonar Obstructiva Crónica (EPOC): Visión global y continuidad de cuidados. Madrid, vol.20, N.7, pp. 337-339. 2003.

2. Estratificación pronóstico en los pacientes con EPOC agudizada que requieren hospitalización. Más humo que señales. Arch Bronconeumol.; 43(11):640-1. 2007.

3. Sobradillo $V$, Miravitlles $M$, Jiménez $C A$, Viejo JL, Masa JF et al. "Estudio IBERPOC en España": Prevalencia de síntomas respiratorios habituales y de limitación crónica al flujo aéreo. Arch Bronconeumol 1999; 35:159-166.

4. Cazzola M, Matera MG and ODonnell K.M. Controversias en el tratamiento farmacológico de la EPOC, association OF B2 - adrenergie agonist and tiotropium is the combination justified. Justified. Arch Bronconeumol 2005; 41 (supl 2):24-31.

5. Grupo de trabajo de la Asociación Latinoamericana del tórax (ALAT). Actualización de las recomendaciones ALAT, sobre exacerbación infecciosa de la EPOC, Arch bromconemol 2004; 40(7):315-25
6. Agusti A, Cello B. Enfermedad pulmonar obstructiva crónica. Led. Masson 2005:328. ISBN:844581556-3

7. Solano $S$, Jiménez CA. Manual del tabaquismo. 2da ED, Masson, 2003: 337-339, 2212.

8. Ancochea Bermudez, J.Girón R.M..Ruiz M.A. Atrapamiento aéreo en la EPOC, el gran olvidado, Tiotropio: análisis crítico. Madrid. Arch _Bronconeumol. 2005; 41(Supl 3):39-47.

9. Grupo de trabajo de la Sociedad Española de Neumología y Cirugía Torácica y de la Sociedad Española de Medicina Familiar y Comunitaria. Recomendaciones para la atención al paciente con enfermedad pulmonar obstructiva crónica. Arch Bronconeumol 2001; 37: 269-278.

10. Escarabill J, Naveran K. Enfermedades Respiratorias. En: Martín Zurro A. Compendio de Atención Primaria. Barcelona: Harcourt; 2000. p. 365-381.

11. Grupo de trabajo de la Asociación Latinoamericana del Tórax (ALAT). Recomendaciones ALAT sobre neumonía adquirida en la comunidad. Arch Bronconeumol 2001; 37:340-8

12. Miravitlles $M$, Fernández $\mathrm{I}$, Guerrero $\mathrm{T}$, Murio $\mathrm{C}$. Desarrollo y resultados de un programa de cribado de la EPOC en atención primaria. El proyecto PADOC. Arch Bronconeumol 2000; 36: 500-505.

13. Miravitlles $M$, De la Rosa $C$, Naberan K, Lamban M, y col. Attitudes Toward the diagnosis of chronic obstructive pulmonary disease in primary care. Arch Bronconeumol, 2006; 42(1):3-8.

14. World health report. Ginebra: World Health Organization, 2000. Disponible en: http://www.who.int/whr/2000/en/statistics.htm.

15. Buhland R, Farmer S. Terapia Farmacológica para futuras exacerbaciones de la Enfermedad Pulmonar Obstructiva Crónica y Asma. Proceeding Am Torca Soc 1:I36-142. 2004.

16. Ferguson GT, Enright PL, Buist AS, Higgins MW. Office spirometry for lung health assessment in adults: a consensus statment from the National Lung Health Education Program. Chest 2000; 117:1146-1161.

17. Jones $\mathrm{P}$, Soler JJ. Eficacia de un programa especifico para pacientes con EPOC que presentan frecuentes agudizaciones. Arch Bronconeumol; 42(10):501-508. octubre 2006. ISBN: 1579-2129

18. Lucas RP, Díaz $M$, López $M$, Rodríguez JM. Enfermedad pulmonar obstructiva crónica: normativas, guías, vías clínicas. Arch Bronconeumol; 40(suple 6):9-15. 2004. 
19. Martin JM. Viejos y nuevos criterios para clasificar la enfermedad pulmonar obstructiva crónica: Arch Bronconeumol; 40(Suple 6):9-15. 2004

20. Eaton T, Withy S, Garrett JE, Mercer J, Whitlock RM, Rea $\mathrm{HH}$. Spirometry in primary care practice: the importance of quality assurance and the impact of spirometry workshops. Chest 1999; 116:416-423

21. Martín Zurro A, Cano Pérez JF. Atención Primaria.: conceptos, organización y práctica clínica. Harcourt Brace. Madrid 2000.

22. Miguel DJ, Izquierdo JA, Molina Paris JM, Bellon CJ, y col. Factores determinantes de la prescripción farmacológica de los pacientes con EPOC estable. Resultados de un estudio multicéntrico español (IDENTEPOC). Arch Bronconeumol. 2005; 45(2):6370.

23. Sin DD, McAlister FA, Man SF. Anthonisen NR. Tratamiento Contemporáneo de la enfermedad Pulmonar Obstructiva Crónica. Review. JAMA. 2003; 290:2301-2312
24. Fernández VA, Bujalance ZM, Leiva FF, Matos CF, García y Col. Utilización de servicios, medidas preventivas y educación sanitaria de los pacientes diagnosticados de enfermedad EPOC en atención primaria (AP) pulmonar obstructiva crónica, 2003.

25. Barberà JA, Peces-Barba G, Agustí AGN, Izquierdo JL, Monsó E, Montemayor T y col. Guía clínica para el diagnóstico y el tratamiento de la enfermedad pulmonar obstructiva crónica. Arch Bronconeumol 2001; 37: 297-316.

Recibido: 07/08/2011

Aceptado para publicación:24/02/2012 